\title{
Ação do Ômega 3 nas reservas energéticas e responsividade insulínica em ratos diabéticos aloxanizados
}

\author{
Omega 6 action on glycogen reserves \\ and glucose homeostasis in aloxan diabetic rats
}

Carlos Alberto da Silva ${ }^{\mathrm{I}}$

MARCella Damas Rodrigues ${ }^{1}$

BRUNo FERREIRA GONÇALVES E SILVA ${ }^{\mathrm{I}}$

Mariele Almeida Pazotto ${ }^{I}$

Patrícia Carla Paulino BELOTO $^{1}$

${ }^{1}$ Universidade Metodista de Piracicaba, (UNIMEP). Piracicaba/ SP - Brasil.
Resumo Analisou-se a ação do ácido graxo Ômega 3 sobre as reservas glicogênicas e regulação glicêmica de ratos diabéticos aloxanizados. Utilizaram-se ratos machos com idade de 2-3 meses, pesando 180-200g, tratados com ração balanceada e água "ad libitum", os quais foram divididos nos seguintes grupos experimentais: Controle, Tratado com ômega 3 (via oral $85 \mathrm{mg} / \mathrm{Kg}, 7$ dias através de gavagem), Diabéticos aloxanizados e Diabéticos aloxanizados tratados com ômega 3 (via oral 85 $\mathrm{mg} / \mathrm{Kg}, 7$ dias). Após a indução do diabetes, foram realizados os testes de tolerância à glicose (GTT), teste de tolerância à insulina (ITT), dosagem de ácidos graxos livres e determinação de glicogênio muscular e hepático por intermédio de metodologia bioquímica. Foi observado que o tratamento com ômega 3 não modificou a responsividade pancreática, expresso no GTT tanto nos ratos normais quanto nos diabéticos, bem como, não modificou a sensibilidade tecidual à insulina avaliado pelo ITT, em ambas as condições. Quanto à glicemia, constatou-se que em decorrência da ação da aloxana desenvolveu-se hiperglicemia, indicando a implantação do diabetes, condição não revertida pelo tratamento com ômega 3. Na avaliação das concentrações glicogênicas hepáticas e musculares, foram verificadas baixas reservas no grupo diabético e no grupo diabético tratado, indicando incapacidade do ômega 3 em reverter o quadro clínico. A concentração plasmática de ácidos graxos livres mostrou valores elevados no grupo diabético, que também não foram revertidos pelo tratamento com ômega 3. Conclui-se que o tratamento com ômega 3 não foi eficiente em minimizar as alterações metabólicas geradas no diabetes.

Palavras-chave: Diabetes. Ômega 3. Aloxana. Glicogênio.

Abstract The action of omega-3 fatty acid on glycogen reserves and glycemic regulation of alloxanized diabetic rats was analyzed. Male rats 2-3 months of age weighing 180-200g treated with balanced ration and "ad libitum" water, which were divided into the following experimental groups: Control, Treated with omega 3 (oral route $85 \mathrm{mg} / \mathrm{Kg}, 7$ days through gavage), alloxanized diabetics and alloxanized diabetics treated with omega-3 (oral route $85 \mathrm{mg} / \mathrm{kg}, 7$ days). After induction of diabetes, it was performed the glucose tolerance tests (GTT), (ITT), free fatty acids dosage and determination of muscle and hepatic glycogen through 
biochemical methodology. It was observed that omega 3 treatment did not modify pancreatic responsiveness, expressed in GTT in both normal and diabetic rats as well, as it did not change the tissue sensitivity to insulin assessed by ITT, in both conditions. As for glycemia, it was observed that as a result of the action of alloxan, hyperglycemia developed indicating the implantation of diabetes, a condition not reversed by treatment with omega 3. In the evaluation of hepatic and muscle glycogen concentrations, low reserves were observed in the diabetic group and in the treated diabetic group, indicating an inability of the omega 3 to revert the clinical condition. The plasma concentration of free fatty acids showed high values in the diabetic group and was not reversed by treatment with omega 3 . It was concluded that the treatment with omega 3 was not efficient in minimizing the metabolic changes generated in diabetes.

Key-words: Diabetes. Omega 3. Alloxan. Glycogen.

\section{INTRODUÇÃO}

O Diabetes Mellitus é uma doença crônica decorrente da diminuição na relação síntese/produção de insulina pelo pâncreas ou pela resistência à insulina pelos tecidos periféricos. $^{1}$

A insulina é um hormônio secretado especificamente pelas células $\beta$ das ilhotas pancreáticas, a partir do aumento na concentração plasmática de glicose, aminoácidos ou devido à ação de fármacos. A ação insulínica consiste em estimular a síntese de glicogênio hepática e muscular, lipogênese, inibir a lipólise, bem como, ativar a síntese proteica e inibir a proteólise, em suma, o foco da ação se traduz como elevação na captação de glicose por tecidos periféricos. ${ }^{2}$

Quando as células $\beta$ pancreáticas são destruídas por anticorpos ou reduzem sua eficiência secretória, ou ainda, na presença de resistência periférica à insulina, observa-se o surgimento de sintomatologia característica da implantação do diabetes, onde consequentemente ocorre elevação na concentração plasmática de glicose (hiperglicemia), perda de peso, polifagia, poliúria, visão turva, polidipsia, podendo haver complicações agudas que causam risco de vida como, a cetoacidose diabética, síndro- me hiperosmolar e a disfunção e falência de diversos órgãos. Para diagnosticar o diabetes mellitus, utiliza-se avaliar a glicemia no jejum ou o comportamento glicêmico após uma sobrecarga de glicose por via oral, empregando-se o teste conhecido como curva glicêmica. Pode-se acompanhar a existência de episódios de hiperglicemia por meio da avaliação da glicosilação da hemoglobina (HbA1C). ${ }^{3,4}$

Estudos utilizando animais na indução experimental do diabetes tornaram-se indispensáveis, pois, por meio destes é possível permitir o desenvolvimento de novas ações terapêuticas. O aloxano é uma substância química que apresenta ação citotóxica nas células $\beta$ das ilhotas pancreáticas degenerando e, por conseguinte, matando-as, e deixam assim de produzir insulina, tornando o organismo diabético. ${ }^{5}$

Entre os tipos de diabetes, destacam-se o Diabetes Mellitus Tipo 1, em que ocorre destruição das células $\beta$ pancreáticas por anticorpos, originando por consequência a deficiência total de insulina, ${ }^{6,7}$ e o Diabetes Mellitus Tipo 2, que é considerado uma doença que compreende de $90 \%$ a $95 \%$ de todos os casos diagnosticados, e se fundamenta na redução da sensibilidade pancreática e/ou resistência no receptor da insulina., 
Os ácidos graxos Ômega 3 são ácidos graxos insaturados, que são essenciais para o metabolismo, pois não podem ser obtidos pela síntese de novo, no entanto, podem ser adquiridos a partir da ingestão alimentar dos ácidos linoleico. Existem três famílias importantes de ácidos graxos comumente consumidos na dieta: $w-9, w-6$ e w-3, sendo que apenas as duas últimas representam os ácidos graxos essenciais para o organismo. Os ácidos graxos das famílias w- 6 e $\mathrm{w}-3$ competem pelas mesmas enzimas no organismo humano, com isso é necessário atentar-se ao balanço desses lipídeos na alimentação e preconizar a ingestão adequada conforme estabelecido pelo Institute of Medicina, por meio das Dietary Reference Intakes (DRIs), onde, a relação de ingestão atual padrão apresenta os valores de 17:1,6/ w-6:w3. ${ }^{7}$

O balanço dietético de lipídeos é foco de novos estudos que relacionam os tipos de ácidos graxos poli-insaturados ingeridos e sua utilização no controle de respostas inflamatórias. O alto consumo de ácido linoleico $(\omega-6-\omega-3)$ favorece o aumento do conteúdo de ácido araquidônico nos fosfolipídios das membranas celulares, aumentando, por conseguinte, a produção da prostaglandina (PGE2) e leucotrieno (LTB4), por meio das vias enzimáticas da ciclooxigenase (COX) e 5- lipoxigenase (5LOX), respectivamente. ${ }^{9}$ Os ácidos graxos poli-insaturados podem interferir nas interações intracelulares, receptores de membranas, crescimento das células, transporte dos nutrientes e sinais de transdução. ${ }^{10}$

Frente às múltiplas ações ligadas à suplementação com ômega 3, existe a hipótese que esse ácido graxo possa modificar, de forma positiva, os desajustes gerados pela implantação do diabetes mellitus.

\section{Objetivo}

Analisar a ação da suplementação com o ácido graxo Ômega 3 sobre as reservas hepáticas e musculares de glicogênio, sensibilidade pancreática à glicose (GTT), sensibilidade tecidual a insulina (KITT) e concentração plasmática de ácidos graxos livres, em ratos diabéticos aloxanizados.

\section{Metodologia}

Foram utilizados ratos machos adultos, com idade entre 2-3 meses, pesando entre 180 a 200 gramas, tratados com ração balanceada e água "ad libitum", os quais foram divididos em grupos experimentais denominados de Controle (C), Tratado com ômega 3 (T; via oral $85 \mathrm{mg} / \mathrm{Kg}, 7$ dias), Diabéticos aloxanizados (D), Diabéticos aloxanizados tratados com ômega 3 (DT; via oral $85 \mathrm{mg} / \mathrm{Kg}, 7$ dias) todos com $n=10$. A pesquisa foi autorizada pela Comissão de ética em pesquisa envolvendo animais sob o protocolo 10/2012.

Para a indução do diabetes experimental, foi administrado através da via intraperitoneal, uma dose única de $120 \mathrm{mg} / \mathrm{Kg}$ de aloxano (diluído a $2 \%$ em tampão citrato, pH 4,5). Após 6 horas da indução, foi fornecida uma solução de glicose a $10 \%$ para evitar uma hipoglicemia fatal, devido à liberação maciça de insulina que ocorre após a destruição das células beta. No sétimo dia, após a administração da aloxana, a glicose sanguínea foi determinada, sendo que os animais considerados diabéticos apresentavam glicemia superior a $180 \mathrm{mg} / \mathrm{dL}$. 
Os animais foram divididos em grupos e submetidos ao teste oral de tolerância à glicose (GTT), após anestesia com pentobarbital sódico $(40 \mathrm{mg} / \mathrm{Kg})$. Nessa técnica, o sangue foi coletado por meio de um corte na ponta da cauda nos tempos de 0, 30, 60, 90 e 120 minutos. Sendo o bolus de glicose na dose de $2 \mathrm{~g} / \mathrm{Kg}$ administrada após a coleta do tempo zero. A glicemia foi monitorada através do uso de glicoteste. Posteriormente, os ratos foram submetidos ao teste de tolerância à insulina (ITT) e, nessa técnica, após anestesia, insulina foi administrada pela via intraperitoneal na concentração de $2 \mathrm{U} / \mathrm{Kg}$, após o tempo zero. O sangue foi coletado nos tempos de $0 ; 2,5 ; 5 ; 10$ e 15 minutos sendo a glicemia monitorada por intermédio do uso de glicoteste. Para determinação da concentração plasmática de ácidos graxos livres (AGL), foi utilizado o método proposto por Regouw. ${ }^{11} \mathrm{Na}$ avaliação do conteúdo de glicogênio, amostras do fígado e dos músculos sóleo e gastrocnêmio, porção branca, foram digeridas em $\mathrm{KOH} \mathrm{30 \%} \mathrm{a} \mathrm{quente} \mathrm{e} \mathrm{o} \mathrm{glico-}$ gênio precipitado a partir da passagem por etanol e submetido a colorimetria com fenol, metodologia rotineira de laboratório de pesquisa. A análise estatística foi realizada através de ANOVA e teste de Tukey, $\mathrm{p}<0,05$.

\section{RESUltados}

Inicialmente, foi avaliada a ação da aloxana sobre a responsividade pancreática à glicose através do GTT e foi observado no grupo diabético que houve elevação de 208\% na área sob a curva (AUC) indicando comprometimento na responsividade do processo secretório da insulina e instalação da condição hiperglicêmica que acompanha a patologia. No mesmo perfil de análise, foi verificado que o tratamento com ômega 3 não modificou a responsividade pancreática em ratos normais, bem como, não modificou as alterações pancreáticas promovidas pela aloxana e representada na AUC (tabela 1).

No que se refere à sensibilidade tecidual à insulina representado no KITT, foi observado que os ratos aloxanizados desenvolveram o quadro de resistência à insulina, havendo redução de 51,6\% na velocidade de captação da hexose. Nessa análise, foi verificado que o tratamento com ômega 3 não modificou a velocidade de decaimento da glicemia tanto no grupo controle tratado quanto no grupo diabético (tabela 1). No que diz respeito à glicemia, foi visto que em decorrência da ação da aloxana a glicemia basal apresentou-se $125 \%$ acima daquela observada no grupo controle, indicando a implantação do diabetes (tabela 2). Na mesma tabela, pode ser analisado que o tratamento não modificou a glicemia de ratos normais nem dos diabéticos (tabela 2).

A seguir, o estudo foi direcionado à avaliação da concentração plasmática de ácidos graxos livres, sendo verificado que no grupo diabético as concentrações foram elevadas em $123 \%$ e o tratamento com ômega 3 não foi eficiente em modificar essas mudanças metabólicas (tabela 2).

Seguindo a proposta, foram avaliadas as reservas glicogênicas, sendo observado redução significativa e generalizada, em decorrência do diabetes atingindo $43,6 \%$ no fígado, 39,2\% no músculo sóleo e 54,8\% no gastrocnênio, valores compatíveis com descrições na literatura. Nessa análise, foi verificado que o tratamento não modificou as reservas glicogênicas não diferindo se compararmos o grupo tratado com o controle ou o diabético tratado com o grupo diabético não tratado, como pode ser observado na tabela 3 . 
Tabela 1. Área sob a curva (AUC, $\mathrm{mg} / \mathrm{dL} / 120 \mathrm{~min}$ ) e constante de decaimento da glicemia (KITT, \%/min) dos grupos controle (C); tratado com ômega 3 (T); Diabéticos (D); e Diabéticos tratados com ômega $6(\mathrm{DT})$. Os valores correspondem à média $\pm \mathrm{dp}, \mathrm{n}=10 .{ }^{*} \mathrm{P}<0,05$ comparado ao controle.

\begin{tabular}{ccccc}
\hline & C & T & D & DT \\
\hline AUC & $482,5 \pm 23$ & $420 \pm 30$ & $1.490 \pm 31$ & $1350 \pm 21$ \\
\hline KITT & $10,15 \pm 0,2$ & $9,98 \pm 0,3$ & $4,91 \pm 0,2^{\star}$ & $5,20 \pm 0,3^{\star}$ \\
\hline
\end{tabular}

Tabela 2. Concentração plasmática de glicose $(\mathrm{mg} / \mathrm{dL})$ e ácidos graxos livres $(\mathrm{mmol} / \mathrm{L})$ dos grupos controle (C); tratado com ômega 3 (T); Diabéticos (D); e Diabéticos tratados com ômega 3 (DT). Os valores correspondem à média $\pm \mathrm{dp}, \mathrm{n}=10 .{ }^{\star} \mathrm{P}<0,05$ comparado ao controle.

\begin{tabular}{lllll}
\hline & C & T & D & DT \\
\hline Glicose $(\mathrm{mg} / \mathrm{dL})$ & $118.47 \pm 1.9$ & $121,57 \pm 2.8$ & $267.24 \pm 20^{\star}$ & $262,37 \pm 12^{\star}$ \\
\hline AGL $(\mathrm{mmol} / \mathrm{L})$ & $0.39 \pm 0.03$ & $0.37 \pm 0.02$ & $0.87 \pm 0.04^{*}$ & $0.88 \pm 0.03^{*}$ \\
\hline
\end{tabular}

Tabela 3. Concentração de glicogênio (mg/100mg) de diferentes estruturas dos grupos controle (C); controle tratado com ômega 3 (T); Diabéticos (D); e Diabéticos tratados com ômega 3 (DT). Os valores correspondem à média $\pm \mathrm{dp}, \mathrm{n}=10 .{ }^{\star} \mathrm{P}<0,05$ comparado ao controle

\begin{tabular}{llll}
\hline & FÍGADO & SÓLEO & GASTROCNÊMIO \\
\hline $\mathrm{C}$ & $3.80 \pm 0,2$ & $0,28 \pm 0.04$ & $0,31 \pm 0.02$ \\
\hline $\mathrm{T}$ & $3.66 \pm 0,1$ & $0,25 \pm 0.04$ & $0,32 \pm 0.01$ \\
\hline $\mathrm{D}$ & $1.69 \pm 0.1^{*}$ & $0,17 \pm 0.03^{*}$ & $0,14 \pm 0.01^{*}$ \\
\hline $\mathrm{DT}$ & $2.00 \pm 0.3^{*}$ & $0,18 \pm 0.02^{\star}$ & $0,17 \pm 0.07^{\star}$ \\
\hline
\end{tabular}

\section{Discussão}

O diabetes é uma doença de cunho endócrino e metabólico decorrente de uma deficiência relativa ou absoluta de insulina, comprometendo o metabolismo de carboidratos, gorduras e proteínas. ${ }^{12}$ Nessa condição, as ilhotas pancreáticas secretam uma menor quantidade de insulina, ou os tecidos periféricos tornam-se resistentes. Na literatura, estudos básicos são importantes para a compreensão dos mecanismos fisiopatológicos do diabetes, bem como, para a sua prevenção e tratamento. ${ }^{13}$
Há muitos modelos experimentais indicados para a indução do diabetes existindo vários agentes químicos citotóxicos para as células $\beta$ do pâncreas, porém apenas a aloxana e a estreptozotocina têm sido sistematicamente investigadas e amplamente empregadas para induzir diabetes em animais de experimentação. ${ }^{14} \mathrm{~A}$ aloxana é uma substância com propriedades diabetogênicas com ação seletiva e destrutiva das células $\beta$ pancreáticas, levando à degeneração $\mathrm{e}$ morte definitiva das mesmas. ${ }^{15}$ Nosso trabalho mostra diferentes parâmetros que apontam o sucesso da indução do diabetes pela 
aloxana e o subsequente comprometimento no processo secretório, como a hiperglicemia e a maior área sob a curva no teste de tolerância à glicose.

Estudos clássicos demonstraram o aumento glicêmico, após a aplicação do aloxana. ${ }^{16}$ Dessa forma, no presente estudo observamos elevação na glicemia em períodos similares aos descritos na literatura, ou seja, nas 8 horas subsequentes à injeção da aloxana. ${ }^{17}$ Um ponto merecedor de destaque é que o tratamento com ômega 3 não foi eficiente para minimizar os eventos deflagrados pela aloxana, representado por diminuição na quantidade de retículo endoplasmático rugoso, das mitocôndrias e do complexo de Golgi, uma vez que o processo secretório da insulina induzido pela glicose foi comprometido. ${ }^{16}$

No que se refere à sinalização insulínica, sabe-se que as ações iniciam-se pela ligação do hormônio ao seu receptor na membrana plasmática, o qual apresenta uma subunidade alfa, posicionado em direção ao extracelular e confere alta afinidade de ligação da insulina ao receptor e uma unidade beta que se autofosforila, transferindo ao sinal para a face citosólica. ${ }^{2}$ Foi verificado que os grupos diabéticos apresentaram redução na constante de decaimento da glicemia obtido no teste de tolerância à insulina, indicando o status de resistência, representado pela redução na atividade das vias enzimáticas citosólicas do receptor e pelas subunidades do receptor da insulina ou IRSs. ${ }^{18} \mathrm{O}$ IRS-1 é uma proteína crucial no processo de transmissão do sinal insulínico, localizada estrategicamente na fase inicial dessa sinalização. Atua como proteína "ancoradouro" que ativa diversas enzimas, sendo provavel- mente um ponto de ramificação dos efeitos pleiotrópicos da insulina. Portanto, este estudo, ao verificar redução na velocidade de captação da hexose, sugere que o ômega 3 não reverte as alterações diabetogênicas da aloxana.

Tendo em vista que algumas vias metabólicas de transmissão do sinal insulínico têm papel determinante na homeostase da glicose, o estudo foi direcionado à avaliação das principais reservas energéticas, sabendo-se que existe uma associação entre as IRSs com sistemas enzimáticos citosólicos, como a PI-3-quinase, cuja atividade é essencial para induzir elevação no transporte de glicose em tecido muscular esquelético. ${ }^{19,20}$ Vários estudos demonstram uma regulação tecido-específica dos níveis e grau de fosforilação do IRS-1 quando analisado em fígado ou músculo.

Como reflexo das alterações provocadas nas ilhotas pancreáticas, foi observado redução nas reservas glicogênicas tanto hepáticas quanto musculares, fato que indica modificação na homeostasia das vias insulínicas nos tecidos periféricos alvo do hormônio. O estudo da avaliação das reservas glicogênicas hepáticas e musculares também não mostra efeito significativo do tratamento com ômega 3 em superar o status diabetogênico.

Com relação ao ômega 3 não apresentar ação expressiva sobre as reservas glicogênicas, acompanhamos recente proposta onde foi sugerido que a suplementação com ômega 3 não altera a glicemia nem o metabolismo de carboidratos no fígado de humanos diabéticos. ${ }^{19}$ 


\section{CONSIDERAÇÕES FINAIS}

O tratamento com ômega 3 não foi eficiente em recuperar as reservas glicogênicas hepáticas e musculares comprometidas pelo diabetes e não houve melhora na constante de captação de glicose representado pelo baixo KITT, nem na sensibilidade das células beta pancreáticas frente à administração de sobrecarga de glicose. Cabe ressaltar que o tratamento não influenciou na intensa lipólise, visto as altas concentrações plasmáticas de ácidos graxos livres. Em suma, a su- plementação com ômega 3 não exerce papel curativo quando a condição diabetogênica está implantada.

\section{Agradecimento}

Ft. MSc. Marcella Damas Rodrigues ( in memoriam ). Você será lembrada como uma profissional que nunca lhe faltou perseverança, competência, integridade e amizade. Temos ótimas lembranças da pessoa especial que sempre foi para todos nós. Muito obrigado por ter sido uma importante pesquisadora no nosso grupo de pesquisa e de ter passado pela nossa vida.

\section{REFERÊNCIAS}

1. OMS, 2011.

2. Carvalheira JBC, Zecchin HG, Saad MJA. Vias de Sinalização da Insulina. Arq Bras Endocrinol Metab. 2002; 46/4:419-425.

3. Gross, JL. Diabetes Melito: Diagnóstico, Classificação e Avaliação do Controle Glicêmico. Arq Bras Endocrinol Metab, 2002; 46 (1): 32-36.

4. Negri G. Diabetes melito: plantas e princípios ativos naturais hipoglicemiantes. Revista Brasileira de Ciências Farmacêutica, 2005; 41 (2): 121-142.

5. Zhou Y., Tian C., Jia C. Association of fish and n-3 fatty acid intake with the risk of type 2 diabetes: a meta-analysis of prospective studies. Br J Nutr. 2012; 108: 408-417.

6. Fernandes, APM et al. Fatores imunogenéticos associados ao diabetes mellitus do tipo 1. Rev. Latino-Am. Enfermagem. Ribeirão Preto, 2005; 13 (5): 12-17.

7. Garofolo A., Petrilli AS. Balanço entre ácidos graxos ômega-3 e 6 na resposta inflamatória em pacientes com câncer e caquexia. Rev. Nutr. Campinas, 2006;19 (5): 136-141.

8. Krause J. Alimentos, Nutrição e Dietoterapia. Elsevier Medicina Brasil - Grupo Elsevier, 2010.

9. Calder PC. Long-Chain N-3 Fatty Acids and Inflammation: Potential Application in Surgical and Trauma Patients. Braz. J. Med. Biol. Res. 2003; 36 (4): 433-46.

10. Montori VM, Farmer A., Wollan PC, Dinneen SF. Fish oil supplementation in type 2 diabetes: a quantitative systematic review. Diabetes Care. 2000; 23(9): 1.407-15.

11. Regouw BJM, Cornelissem PJH, Helder R., Spijkers JBF, Weeber YMM. Specific determination of the free fatty acid in plasma. Clin Chin Acta. 1971; 31(1): 187-95.

12. Cai Chen, Xuefeng Yu, Shiying Shao. Effects of Omega-3 Fatty Acid Supplementation on Glucose Control and Lipid Levels in Type 2 Diabetes: A Meta-Analysis. PLoS One. 2015; 10(10): e0139565.

13. Cesaretti MLR, Kohlmann OJR. Modelos experimentais de Resistência a Insulina e Obesidade: Lições Aprendidas. Arquivos Brasileiros de Endocrinologia e Metabologia, 2006; 50 (2): 190-197.

14. Pelikanova T., Kohout M., Valek J., Kazdova L., Base J. Metabolic effects of omega-3 fatty acids in type 2 (non-insulin-dependent) diabetic patients. Ann N Y Acad Sci. 1993; 683: 272-278. 
15. Assis AM, Rech A., Longoni A., Rotta LN, Denardin CC, Pasquali MA. $\Omega 3$-Polyunsaturated fatty acids prevent lipoperoxidation, modulate antioxidant enzymes, and reduce lipid content but do not alter glycogen metabolism in the livers of diabetic rats fed on a high fat thermolyzed diet. Mol Cell Biochem. 2012; 361(1-2): 151-60.

16. Lenzen S. The mechanisms of alloxan - and streptozotocin - induced diabetes. Diabetologia. Berlin, 2008; 51:216-226.

17. Leme JACA, Castellar A., Remedio RN, Barbosa RA, Moura LP, Dalia RA. Efeitos em curto prazo da aplicação de aloxana para Indução de diabetes em ratos wistar. Bioscience Journal, 2010, 26 (3): 451-456.

18. Saad MJA, Zecchin HG, Carvalheira JBC. Vias de Sinalização da Insulina Arq Bras Endocrinol Metab. 2002; 46 (4): 202-206.

19. Saad MJA. Molecular mechanisms of insulin resistence. Braz. J. Med. Biol. Res. 1994; 27: 941-957.

20. Muley A., Muley P., Shah M. ALA, fatty fish or marine n-3 fatty acids for preventing DM?: a systematic review and meta-analysis. Curr Diabetes Rev. 2014; 10: 158-165.

\section{DAdos dos Autores}

\section{Carlos Alberto da Silva}

Doutor em Ciências Fisiológicas pela Universidade Estadual de Campinas. Professor no Programa de Pós-Graduação em Ciências do Movimento Humano na Universidade Metodista de Piracicaba. Piracicaba/SP - Brasil. casilva@unimep.br

\section{Marcella Damas Rodrigues}

Mestre em Fisioterapia pela Universidade Metodista de Piracicaba. in memoriam. Piracicaba/ SP Brasil.

\section{Bruno Ferreira Gonçalves e Silva}

Graduando do curso de Medicina Veterinária pela Universidade Paulista. Campinas/SP - Brasil. bruninho0201@hotmail.com

\section{Mariele Almeida Pazotto}

Graduada em Ciências Biológicas pela Universidade Metodista de Piracicaba. Piracicaba/ SP - Brasil. marielepazotto@yahoo.com.br

\section{Patrícia Carla Paulino Beloto}

Graduada em Ciências Biológicas pela Universidade Metodista de Piracicaba. Piracicaba/ SP - Brasil. pcpaulin@unimep.br

Submetido em: 6-8-2013

Aceito em: 5-4-2017 